\title{
LL-ADR: Action Design Research in Living Labs
}

\author{
Tanguy Coenen \\ iMinds - SMIT - Vrije \\ Universiteit Brussel \\ tanguy.coenen@iminds.be
}

\author{
Vincent Donche \\ University of Antwerp - \\ Institute for Education and \\ Information Sciences \\ vincent.donche@uantwerpen.be
}

\author{
Pieter Ballon \\ iMinds - SMIT - Vrije \\ Universiteit Brussel \\ pieter.ballon@iminds.be
}

\begin{abstract}
Design Science research is a mainstream Information systems discipline, yet Living Lab literature seems not to leverage its insights. The paper describes how Living Labs can be conducted as Design Science Research (DSR) by adapting the Action Design Research method. The resulting method is named LL-ADR. A case is presented to indicate how LL-ADR can be applied and findings for both Living Labs and DSR are discussed.
\end{abstract}

\section{Introduction}

Living labs are popular as a system for conducting innovation-driven research, yet the literature on how to do academic research in Living Lab projects is relatively scant and heterogeneous. Still, many Living Lab projects are carried out in part by academic institutions. In academic research settings, the goals of a Living Lab seem congruous with the role of Design science research (DSR), where "knowledge and understanding of a problem domain and its solution are achieved in the building and application of the designed artifact."([5] p75).

The DSR field has recently flourished and has been accepted as mainstream in information systems research counting many high-quality methodological publications. However, the Living Lab community seems to have passed up on the opportunity to leverage the insights of DSR. This paper aims to offer a first effort to remedy this by grounding Living Lab projects in the information systems research paradigm that is DSR. The scope of the paper is limited to Living Labs that aim to create software innovations, as DSR is an information systems discipline and does not claim to be valid for other design activities. In addition, the findings of this paper are limited to Living Labs of which some of the stakeholders have as a goal to build academic knowledge through the creation of an information system artifact.
The structure of this paper is as follows. First, we introduce the DSR literature that is particularly relevant to Living Labs, after which we discuss Living Labs themselves. Then, we look at the different available Living Lab methods and discuss how they fit in Action Design Research, a DSR method. This we call LL-ADR, which is shorthand for Living Lab Action Design Research. Finally, we provide a case study detailing how a Living Lab project showed most of the traits of LL-ADR.

\section{Design science research and Action Research}

Hevner et al [5] posit that the central goal of DSR is to produce knowledge that can be used to create future versions of a certain class (i.e. type) of information system (IS). This is done by building and evaluating prototypes that represent instances of the IS class. Both the design and the evaluation need to be done in a rigorous way, meaning that the existing knowledge base must be closely analyzed to be able to frame both the design process and it's results in terms of its innovative value and that appropriate evaluation methods need to be applied.

As DSR often aims to leverage practical design projects to perform actions that allow research, it has been debated ([9],[6]) if Action Research methodology can be a suitable methodology for doing DSR. Livari \& Venable [9] define DSR as "a research activity that invents or builds new, innovative artifacts for solving problems or achieving improvements, i.e. DSR creates new means for achieving some general (unsituated) goal, as its major research contributions. Such new and innovative artifacts create new reality, rather than explaining existing reality or helping to make sense of it." ([9] p3). Their stance is that, although DSR and AR are quite distinct, DSR is a research orientation that can use various research methods, among which Action Research (AR). However, when DSR and AR are used in union, researchers should be aware of the fact 
that new technology is being developed on the fly and often exposed to the societal context in a way that can be largely untested and possibly unstable. This presents significant risks to the stakeholders of the project, as their normal functioning can be disrupted by technological failure.

As a method for combining DSR and AR, Sein et al [15] proposed Action Design Research (ADR), which is composed of the following steps: 1 . Problem formulation, 2. Building, intervention and evaluation, 3 . Reflection and learning and 4. Formalization of learning. Peffers et al [11] proposed a method for DSR that is very similar to ADR: 1. identify problem, 2. define solution objectives, 3. design and development, 4. demonstration, 5. evaluation and 6. communication. However, according to Sein et al [15], approaches like Peffers [11] are constructed too much like stage-gate models and fail to grasp the nature of the DSR artifact as an ensemble, shaped by both its designers and the organizational context in which it is utilized. It is this interplay between design and context that also lies at the heart of Living Labs. As we will see, AR had been proposed as a method for Living Lab design projects. Due to $\mathrm{AR}$ as a common denominator, it seems appropriate to expect a relationship between ADR and Living Lab projects.

\section{Living Labs}

There are many definitions of the Living Lab concept (e.g. [12] [19]). We use the one proposed by Westerlund and Leminen [19] because it encompasses all relevant aspects in a clear way. Living Labs are thus seen as "physical regions or virtual realities with stakeholders from publicprivate-people partnerships (4Ps) of firms, public agencies, universities, institutes, and users all collaborating for creation, prototyping, validating, and testing of new technologies, services, products and systems in real-life contexts." ([19], p20). Two main characteristics are salient in this definition: research in the wild and the 4P stakeholders.

As indicated by Almirall et al [1], "The most distinctive characteristic of Living Lab methodologies is the focus on real-life, "in the wild" environments as the locus of research." ([1], p15). Such research allows the researchers to find new meanings, capture tacit user knowledge and validate the ecosystem around the artifact.

The 4P aspect of the definition indicates that most Living Labs feature a mixture of commercial and non-commercial goals, as both commercial and noncommercial actors are a part of the project. In addition, "people", i.e. representative end-users are part of the project so they can provide the researchers with the knowledge they need to create systems that are relevant to the target end-user group.

Leminen et al [8] describe four types of Living Labs: utilizer-driven, enabler-driven, provider-driven and user-driven. Utilizers are companies that use Living labs to develop and test their products. Enablers are public-sector agents and nongovernmental organizations that aim to achieve societal improvement through Living Labs. Providers are knowledge institutions like universities or consultancy agencies that aim to engage in knowledge creation through the Living Lab. Finally, user-driven Living Labs are initiated by user communities and are geared toward solving everyday user-related problems. Of these four different types of Living Labs, only the provider-driven Living Lab is concerned with academic knowledge and theory production. As such knowledge production is one of the main DSR objectives, it seems evident that DSR will be in the first place applicable to Living Lab projects that do engage in knowledge production. We therefore limit the scope of the findings in this paper to provider-driven Living Lab projects.

\section{Methods for performing Living Labs}

The literature on how to undertake Living Lab projects is limited. However, some attempts have been undertaken. Schaffers et al [13] already proposed that the work on the investigation of information systems through Action Research is a natural fit for guiding Living Lab methodology. A 5phase methodology was proposed to do this, composed of the following stages: diagnosing, action planning, action taking, evaluation and learning [13]. Pierson and Lievens [12] defined a Living Lab method that comprises the following phases: contextualization, concretization, implementation and feedback. Bergvall-Kåreborn et al [1] proposed the FormIT Living Lab method, which is also based on Action Research. They described a cyclical approach, composed of the following cycles: generate needs, design concepts and evaluate concepts. There are three types of such cycles, applied on increasingly more mature prototype types: concepts, prototypes and final systems. Tang \& $\mathrm{Ha}$ ma la inen [17] propose a five-step method, with the following phases: vision, prototype, evaluation, diffusion and adoption. This overview of Living Lab methods allows us to investigate the methodological fit between ADR and Living Lab projects. 


\section{Methodological fit between ADR and Living Lab projects}

As shown in Table I, the individual steps in the four available Living Lab methods described above can be bundled in four different methodological groups, allowing us to summarize the available literature on Living Lab methods by means of an adapted version of the ADR phases. The grouping is as follows:

Problem formulation phase: bundles diagnosing, contextualization, generate needs, vision.

Building, intervention, evaluation and learning phase:

- Build \& intervene: bundles action planning, action taking, concretization, implementation, design concepts and prototype

-Evaluate: bundles evaluate concepts and evaluation,

Table I: Stages in the different Living Lab methods. Shades of grey indicate stages that can be grouped under a single ADR label. From left to right: problem formulation; build and intervene; evaluate; learn

\begin{tabular}{|l|l|l|l|l|l|}
\hline Living Lab method & \multicolumn{5}{|c|}{ Phase } \\
\hline Schaffers et al (2008) [13] & Diagnosing & Action planning & Action Taking & Evaluation & Learning \\
\hline Pierson \& Lievens (2005) [12] & Contextualization & Concretization & Implementation & Feedback & \\
\hline Bergvall-Kåreborn et al (2006) [1] & Generate needs & Design concepts & Evaluate concepts & & \\
\hline Tang \& Hämäläinen (2012) [17] & Vision & Prototype & Evaluation & Diffusion & Adoption \\
\hline
\end{tabular}

The building, intervention and evaluation (BIE) concepts are bundled under a single label in ADR because they occur concurrently. Indeed, building takes place during the intervention, i.e. the deployment of the prototype in a real-world environment. Very often in Living Labs, the intervention takes place over a longer period and therefore necessitates continuous building in the form of bug-fixes and improvements. Similarly, evaluation occurs together with building as well as intervention, as frequently in Living Labs, in the wild trials are organized in which the evaluation takes place through observation of the end-users.

In this paper, the build, intervention and evaluation phase has been merged with ADR's reflection and learning phase in Table I. Indeed we find that in Living Labs projects, the learning and reflection takes place as concurrently as the build, intervene and evaluation processes. In the practical settings that constitute Living Labs, one does not learn as a pre-meditated timed activity, but learns while conduction the project. Therefore, we renamed this stage to BIEL, for build, intervene, evaluate and learn.

The diffusion and adoption phases by Tang \& Ha ma la inen [17] do not map to the ADR method, because ADR is about research through the creation of prototypes. As prototypes are typically
- Learning: bundles feedback and learning

Formalization of learning phase: is not a part of current Living Lab methods. Although elements of learning phase are included in two of the four methods, the actual formalization of this learning as is proposed in ADR is never described.

That ADR's formalization of learning phase does not map to any phase in the available Living Lab methodologies indicates that Living Labs are seldom conducted as DSR. Indeed, it is essential to DSR that what has been learned is formalized in a way that will inform the creation of future instances of the class of systems that were defined in the problem formulation phase. It is therefore important that this phase becomes a more integral part in salient Living Lab methods, if Living Labs are to gain more traction as a scientifically valid tool. not meant to be brought to market, these phases seem to go beyond the research scope of DSR. This again means that ADR is more applicable to providerdriven Living Lab projects, that aim at the creation of academic knowledge, than to other types.

\section{LL-ADR: Conducting Living Labs with ADR}

Now that a fit between Living Lab methods and ADR has been established, it is possible to learn from the available Living Lab and DSR methods how to perform Living Lab flavored ADR. The DSR literature can be of help, as ADR is a subset of DSR. We call the resulting method LL-ADR, representing the ADR method, applied in a Living Lab context.

As a general remark, it is important to point out a difference regarding the context in which ADR and Living Labs are conducted. ADR and much of the DSR literature in general refers to the organization as the context in which the research occurs. However, Living Labs are often not conducted within the boundaries of an organization, but in a societal context. Organizations of different kinds are very often part of the project, but the interventions take place outside of their own limits. For example, many Living Lab projects are conducted in cities, featuring a mix of stakeholders like citizens, civic 
organizations, local SME's and the city government. As a result of this difference in implementation context, we will substitute ADR's references to organizations by society in the discussion of LLADR, especially regarding the naming of the phases that are derived from ADR.

LL-ADR contains four phases, each containing a number of tasks and principles derived from ADR. Table II provides an overview of the tasks and principles, ordered per LL-ADR phase. We will briefly include them here and will further discuss them later, in the case study.

\subsection{Problem formulation phase}

DSR specifies that the research output can be in the form of constructs, models, methods, implementations and design principles. Focusing on such contributions will allow the Living Lab researcher to be aware of the type of outcome that can be realized at the end of the project. It is especially important to keep these elements in mind during the execution of the project, as many of the possible contributions to the state of the art will only become apparent during the course of the project. This is different from other scientific disciplines, where the problems to be investigated are known upfront and where the hypotheses to be tested should not change. In DSR, new aspects of the problem definition can surface at any time. Gill \& Hevner [4] illustrate this problem through analogy with a puzzle: "At the beginning of a puzzle, as in a design process, you have a collection of pieces that can only be put together in certain ways. True "problems" - in the form of missing pieces - tend to be discovered near the end of the assembly, when the gaps are identified." ([4], p19).

6.1.1 Tasks It is not always explicitly recognized by Living Lab practitioners that a research question lies at the heart of a project. However, as indicated by Newell and Simon (1976), the creation of new IS artifacts can always be seen as an experiment that poses a question to nature. Making sure that this question or set of questions is made explicit and is referred to can improve the project's focus. Especially in Living Lab projects in which the aims are very broad (e.g. increase the engagement of inhabitants with the city, like in the ZWERM project that will be described later), having a more focused problem formulation is useful. Casting the research questions as an instance of a class of problem allows a more focused literature study as well as later contributions of design knowledge to the state-of-the art.
The theoretical bases and related technological predecessors must be identified and studied, to make sure that the wheel is not reinvented and to guide the design process.

Another task to be undertaken in the problem formulation phase is securing the commitment of the participating stakeholders in the longer term [15]. This is especially relevant to Living Lab projects, as they involve a broad set of stakeholders. In Living Labs, such commitment can be achieved by making sure that the interests of all the participating stakeholders are addressed by the problem formulation, i.e. that the research scope is mutually agreed on by all Living Lab stakeholders.

Finally, the proper roles and responsibilities need to be established. Nyström et al [10] have identified 17 roles that are typical to Living Labs: webber, instigator, gatekeeper, advocate, planner, producer, accessory provider, coordinator, builder, messenger, facilitator, orchestrator, integrator, informant, tester, contributor and co-creator. One person can play multiple roles. This typology can be used to check if the right responsibilities have been attributed to the right people. However, we notice that there is no role which coincides with the activities that are typical to DSR. Therefore, in the context of LL-ADR, we add the role of design researcher to carry out the tasks that give DSR it's scientific grounding, which are most related to the formulation of research questions, the collection of data and the formalization of learning.

6.1.2 Principles The practice-inspired research principle urges researchers to see field problems as knowledge creation opportunities. This can be done by aiming to contribute to the design knowledge related to the class of problems that must be identified in the problem formulation phase. Doing this is highly relevant to Living Lab practice, where leveraging the design project for design research purposes is often neglected.

The theory-ingrained artifact phase underlines that the artifacts that are built during the project are informed by theories. Such theories are essential in structuring the problem, identifying solution possibilities and guiding design [15].

\subsection{Building, intervention, evaluation and learning (BIEL) phase}

The BIEL activities do not necessarily revolve around working clickable prototypes in a summative mode, but also apply to more preliminary prototypes, like story boards, paper prototypes or high-fidelity interface mock-ups, in a formative mode. In Living 
Lab projects, the aim will typically be to involve the end-user as much as possible with the creation and

evaluation of the prototypes in the formative BIEL cycles.

Table II: Tasks and principles in the various LL-ADR phases

\begin{tabular}{|c|c|c|}
\hline Phase & Tasks & Principles \\
\hline Problem formulation & $\begin{array}{l}\text { Identify and conceptualize the research } \\
\text { opportunity; Formulate initial research } \\
\text { questions; Cast the problem as an instance of a } \\
\text { class of problems; Identify contributing } \\
\text { theoretical bases and prior technology } \\
\text { advances; Secure long-term organizational } \\
\text { commitment; Set up roles and responsibilities }\end{array}$ & $\begin{array}{l}\text { Practice-Inspired Research; } \\
\text { Theory-Ingrained Artifact }\end{array}$ \\
\hline $\begin{array}{l}\text { Building, intervention, } \\
\text { evaluation and learning (BIEL) }\end{array}$ & $\begin{array}{l}\text { Discover initial knowledge-creation target; } \\
\text { Select or customize BIEL form; Execute BIEL } \\
\text { cycle(s); Assess need for additional cycles, } \\
\text { repeat; Reflect on the design and redesign } \\
\text { during the project; Evaluate adherence to } \\
\text { principles; Analyze intervention results } \\
\text { according to stated goals }\end{array}$ & $\begin{array}{l}\text { Reciprocal Shaping; } \\
\text { Mutually Influential Roles; } \\
\text { Authentic and Concurrent } \\
\text { Evaluation; Guided } \\
\text { Emergence }\end{array}$ \\
\hline Formalization of learning & $\begin{array}{l}\text { 1. Abstract the learning into concepts for a class } \\
\text { of field problems; Share outcomes and } \\
\text { assessment with practitioners; Articulate } \\
\text { outcomes as design principles; Articulate } \\
\text { learning in light of theories selected; } \\
\text { Formalize results for dissemination }\end{array}$ & Generalizable outcomes \\
\hline
\end{tabular}

The BIEL phase will therefore occur in an iterative mode, creating prototypes that evolve more and more towards functional prototypes, from concepts to prototypes to functional systems [1].

Sein et al [15] describe a continuum with ITdominant $\mathrm{BIE}$ at one end and organization-dominant BIE at the other end. In IT-dominant BIE, the goal is the creation of a specific innovative IT design and stakeholders have the opportunity to shape the artifact during a number of interventions. In organization-dominant BIE, organizational innovation is the main objective. Still, as explained before, Living Labs do not necessarily take place in organizations, but more often than not take place in the broader context of society at large. Therefore, we think it is more accurate to refer to society-dominant BIE than organizational-dominant BIE when positioning LL-ADR.

6.2.1 Tasks The selection of the BIEL form should be done based on the objective of the knowledgecreation target. Is this driven by a need to develop and test a specific new technology? In this case, the BIEL is IT-dominant. However, if the aim is to make societal changes and use IT to achieve these, then the BIEL is society-dominant. In many Living Lab projects, the aim will be societal and there will be a great reliance on user participation in the early phases of the project.

The execution of the BIEL cycles lies at the center of a project and constitutes the richness that can be found in the design, development, evaluation and learning activities. They are carried out as long as needed. While performing the BIEL phases, it is important to practice permanent reflection on the progress of the project, comparing the progress to the stated goals.

6.2.2 Principles The principle of reciprocal shaping stresses that the IT artifact and the context in which it is designed and deployed highly influence each other. Design researchers should be attentive to the processes through which this happens. Living Lab projects are expected to always abide by this principle, as they have end-user participation at the core of their definition. As such, social shaping of technology [20] and domestication [16] are commonly regarded as important theoretical lenses through which to analyze this reciprocal shaping in Living Lab projects.

The principle of mutually influential roles states that project participants will learn from each other and that the artifact will reflect this learning. In Living Lab projects, there is typically a high degree of learning taking place between the different roles that have been listed above. Also, Living Lab projects are inherently composed of multiple stakeholders. Therefore, such learning will transcend organizational and disciplinary boundaries.

The principle of authentic and concurrent evaluation underlines that evaluation does not take place after building as a separate phase, but that both 
take place concurrently. This is also common in Living Lab projects, where field trials often take place over longer periods of time, allowing end-users to embed the technology in their daily lives. In such situations, it is useful to learn from the way the artifact is experienced and built while deploying new versions based on this learning.

The principle of guided emergence is about being sensitive to signals that indicate the mutual shaping between the context and the artifact. This ongoing and often unanticipated mutation of both technology and society allows the reflection and learning that is central to LL-ADR.

\subsection{Formalization of learning}

This is a phase that is not present in mainstream Living Lab projects and is therefore one of the elements that can be most instructive to Living Lab practice.

6.3.1 Tasks The essence of this phase is the abstraction of what was learned during the BIEL phase. This is done to be able to inform future designers regarding the creation of systems belonging to the class of problems identified in the problem formulation phase. Such can be done by means of design principles and should be done in the light of the selected theories. Dissemination of the results is important through the usual scientific or popular channels. This ensures that future projects can leverage the results of the LL-ADR project.

6.3.2 Principles The principle of generalized outcomes is the only principle in this phase. In Action Research, generalization is always challenging, due to the highly situated nature of the created knowledge. However, the DSR researcher should aim as much as possible to indicate how the problems and solutions that were identified during the project can be generalized to a more abstract class of problems and solutions.

\section{Design fitness in provider-driven Living Labs}

Provider-driven Living Labs, to which this paper applies, should not only be concerned with building knowledge by creating software with a high perceived usefulness. According to Gill, \& Hevner [4], a complementary perspective to newly created artifacts is their ability to survive and evolve into future versions of software systems. A number of aspects have been defined that may inform the creation of artifacts in Living Labs in terms of fitness [4]. Three of these seems particularly interesting to Living Lab research: decomposable, malleable and open.

Decomposable artifacts are constituted of a number of independent building blocks or components that have means to communicate. When an artifact is built in such a way, the chances that a particular component will evolve into new useful future versions increases. If the design is not decomposable, the evolution to more fit versions will be an all-or-nothing affair, which is less likely to occur.

Malleability refers to the ability of the user to mold the artifact to its needs. This allows the artifact to respond to changing environments and user needs. If the artifact is malleable, the odds are higher that it will survive in future generations, as users adopt and tweak it to their needs. An open source content management system like Wordpress or Drupal is an example of such malleability. Through customization, the user can modify the default version of the software into something that fits his needs, in effect creating a new version of the artifact.

Finally, the aspect of openness seems important in increasing the fitness of an artifact. This will make the artifact more reusable and available for modification and analysis, especially benefiting the fitness of the artifact when combined with the decomposability and malleability aspects.

Paying attention to these elements during the course of a Living Lab project will allow the resulting artifact to have a better future. For example, we recently were involved in a project in which some partners wanted to delay the open sourcing of the artifact to the end of the project, which was to be in four years time. However, other team members argued that by open sourcing the embryonical system in the first months of the project, the systems would be able to evolve more quickly and would potentially be able to attract external developers who would evolve the system into new and more useful versions. The design was decomposable, which increase the likelihood that someone external to the team would find something interesting to reuse in the set of components that were being developed in the project.

\section{ZWERM as a LL-ADR case}

ZWERM was an outcome of the SmartIP EU Competitive Innovation Program project and was collaboration between a public sector partner (City of Ghent, Belgium), an academic research partner (iMinds) and a private partner (Alcatel Lucent). A detailed description of the system and its workings 
can be found in [7]. Here, we only focus on the aspects that are relevant to the discussion of LLADR. The Ghent pilot, which resulted in ZWERM, was mainly coordinated by iMinds with a knowledge creation objective. It therefore can be qualified as a provider-driven Living Lab in Leminen et al's [8] categorization. For each of the LL-ADR phases, we will indicate how the tasks and principles were present. As LL-ADR is a new research contribution and was not available at the time the ZWERM project was conducted, this case represents a "retrofitting" of the LL-ADR tasks and principles to the case, similar to Sein et al's [15] use of the Volvo competence management case to support the introduction of ADR.

\subsection{Problem formulation phase}

8.1.1 Tasks The initial impetus for this type of project, sponsored by a European research fund, is the "description of work". This document already contained much of the identification and conceptualization of the research opportunity. We were to address citizen engagement with the city through technology. This topic was too broad to be actionable, which is why we engaged in discussions with the prospective end-users of the project, i.e. urban citizens, by conducting a crowd-sourced ideation phase in which inhabitants of Ghent could propose ideas for an app. The details of this crowdsourcing ideation campaign is described in [14]. Together with a number of focus groups with citizens, this resulted in the following initial research question formulation: "How to consolidate and activate social capital in urban neighborhoods using IT?". The theoretical basis which we selected was a combination of gamification, pervasive games and public space interactions. Our system was thus to be game-based and take place in the public space. Therefore, our initial casting of the instance into a class of problems was "Engagement games in the public space that consolidate and activate social capital in urban neighborhoods".

Long-term societal commitment was secured by making sure that all the members of the project team had their agenda's met. For the research partner, this meant an interesting opportunity for knowledge creation. The industry partner got a way of showcasing their new enabling Internet of Things networking technology and the city administration would be doing a project that would benefit its citizens.

Roles and responsibilities were already apparent from the project proposal's description of work. However, setting up roles and responsibilities could have been done in a more explicit way, which we think would have smoothened the project's course. Several of the 17 LL-ADR roles outlined above were present: advocate, planner, producer, coordinator, builder, facilitator, orchestrator, integrator, informant, tester, contributor and co-creator. However, due to space restrictions, we will not go into detail on who played what role.

8.1.2 Principles The principle of practice inspired research was present through the observation that there are many initiatives that aim to engage citizens at the level of the neighborhood in terms of face-toface events. While there is nothing wrong with such initiatives, there are next to no attempts to leverage pervasive mobile technology to support such initiatives. Still, such technologies hold a substantial potential for engaging users, broadening the demographic reach.

Further supporting the claim that the project was inspired by practice is the fact that the research team formulated the scope of the system after conducting crowd-sourced ideation with citizens as well as focus groups. The societal context in which we operated therefore can be said to have had a great influence on shaping the artifact from the early project stages onward.

ZWERM was constructed along the principle of theory-ingrained artifact: the disciplines on which the initial conception of the system was mainly based were gamification, pervasive games and public space interactions.

\subsection{BIEL phase}

8.2.1 Tasks The selected BIEL form was societydominant BIEL. Indeed, the impetus of the project was to increase the engagement of citizens in cities by means of mobile IT. What type of technology this was to be was not predetermined, so we can say that the main objective was society-driven. It was through the collection of input from stakeholders that represented the societal context (citizens, city government, civic organizations) that the contours of the ZERM system appeared.

We executed three BIEL cycles. During the first BIEL cycle, paper prototypes of the interface were created and tested with representative users. In a next BIEL cycle, a first functional prototype was created and tested with a small group of users to validate the game concepts that were central to the system. The last BIEL cycle contained the creation and testing of the final prototype, during which a summative intervention was conducted in two neighborhoods in Ghent for one month. 277 citizens actively used the 
system. In the two last BIEL cycles, that involved working prototypes, a multitude of research techniques were used, allowing a triangulation of research findings that is typical of Living Lab projects [12].

Before carrying out each of the BIEL cycles, knowledge creation targets were set, although not in a rigorous and formalized way. The first BIEL cycle was formative and concentrated on getting the scope of the application right and providing a user-friendly design of the interface. The second BIEL cycle was also formative and focused on studying core game mechanics of which the efficacy was unknown. The last BIEL cycle was summative, featuring a larger scale "in the wild" trial.

At the end of the two formative BIEL cycles, the necessity for new BIEL cycles was evaluated, which was easy, as the system was no way near finished. The essence of these cycles was to be able to reflect on the design and how to redesign accordingly. This was done by analyzing the intervention results according to the goals of each of the BIEL cycles.

Evaluation of adherence to principles was done continuously with regard to Living Lab principles, i.e. user-involvement and real-world testing. However, there was a relative lack of attention to ADR principles as they are explicitly formulated in [15]. Still, as this case study shows, the ADR principles were nevertheless mostly respected, further supporting the natural fit between Living Lab projects and ADR.

8.2.2 Principles ZWERM closely adhered to the principle of reciprocal shaping, especially due to its deployment in the public space. For example the totem-like structures, placed on public squares, had to comply with laws and regulations. As a result, the look and feel of these structures had to convey a temporary quality, allowing it to comply to a different set of city government rules than the one for permanent structures, which was more stringent. The game masters, who were city personnel, also influenced the system. These were city administration personnel, responsible for contributing game content and for example made the researchers aware of the value of integrating the system with social media for increased engagement by and communication with the players.

During the initial problem formulation and BIEL phases the societal context was able to shape the artifact in a way that was incited and guided by the research team. This influence went beyond the traditional software development requirements analysis as it allowed users to set the scope of the project even before any application concept was created. These are example of influences that were initiated and guided by the researchers.

However, there were also other types of societal influences on the artifact, which aligned with the principle of guided emergence. One instance of this principle was the creation by the participants of different types of notes and posters that were pasted on the public totems. This was done to communicate with other players, by for example asking them to come to a public spot at a certain time and play. Also in line with this principle was the fact that we underspecified the game rules so there would be room for emergent self and social expression by the participants [7]. The main such effects that we observed were the organization of player check-in meetings, the bending of rules by the players and the search for sparrows, which were Internet-of-things sensors and part of ZWERM's network of public space interventions. More information on these emergent mechanisms can be found in [7].

The principle of mutually influential roles describes the need for mutual learning between the different involved roles, outlined above. People played multiple roles, which was often advantageous. For example, the fact that a researcher was also living in one of the neighborhoods in which the system was deployed greatly helped the project. It provided very valuable continuous re-shaping of the artifacts, as well as an important operational beachhead in the neighborhood from which to stage logistic activities.

The people fulfilling these roles had a respectful attitude towards each other. Still, there were more or less open conflicts on more than one occasion. Many such conflicts were about a lack of alignment of strategic agenda. Still, the team worked together from the beginning and was able to offer feedback in all the stages of the project's development. ZWERM's success was largely due to the fruitful collaboration across disciplines and the high motivation of the partners involved.

In ZWERM, the principle of authentic and concurrent evaluation manifested itself through an ongoing development during the interventions. There was constant bug-fixing and fine-tuning of the system as a whole. The game campaigns, which were re-set after each week, proved very useful for experimenting with the game rules, like introducing new types of missions or changing the amount of points one would get for performing an action in the game. As we performed observations of user behavior during the course of the interventions, authentic and concurrent evaluation was a given in our research method.

\subsection{Formalization of learning phase}


8.3.1 Tasks The abstraction of the learning into concepts for the class of problems was done after the last BIEL cycle and took place over a period of a year and a half. Design principles were articulated, among others, in the form of a modular component network approach for urban engagement games [7]. This was done in the light of the theories of gamification, pervasive games and interactions in the public space, which had been selected as relevant during the problem formulation phase. These results were formalized for dissemination and published as a number or articles [7][3] and public lectures. This allowed us to share the outcomes with practitioners.

8.3.2 Principles The principle of generalized outcomes was observed. The class of problems to which we believe ZWERM made a contribution are "Engagement games in the public space that consolidate and activate social capital in urban neighborhoods". With ZWERM, we designed a system in which the social environment was the urban neighborhood. It seems plausible that the game mechanics can be adapted to be used in other social contexts than urban neighborhoods by intervening in their public space. For example, in schools, teams could be constituted of students from different classes to reinforce social cohesion between classes. Another example is larger companies, where different departments operate at the same location yet do not talk to each other. To achieve outcome generalization to such environments, Laureyssens et al [7] proposed an "Interaction amplification framework" based on ZWERM's research data that offers design guidelines on how to create pervasive engagement games as a network of interventions in the public space.

\subsection{Design Fitness}

The technologies (Drupal, Flash, mySQL, php) that we selected for use in ZWERM were relatively decomposable, malleable and open. The more market-oriented project that followed ZWERM and reused its core concepts however did not use any of ZWERM's components, but redeveloped all the components. The chosen architecture (Keynote, node.js, Titanium mobile, mongoDB) was faster and more scalable. In this way, the choice we made was done more in terms of usefulness, which is of recognized influence to fitness [4]. We made sure we did not have to accept tradeoffs for this architecture in terms of decomposability, malleability or openness.

As no technical ZWERM components were reused, the fitness of our final ZWERM prototype can be said to be low. This was in part caused by a specific technology (Adobe Flash) that was no longer supported by enough hardware systems. This type of issue can be seen as an addition to the fitness-utility model, which does not account for compatibility as an aspect influencing the fitness of an artifact. In the evolutionary terminology that the utility-fitness model is formulate in, this would be similar to certain genetic traits not being able to transfer to further generations because of incompatibility. Compatibility seems not to be accounted for by the existing list of influences on an artifact's fitness in [4].

An additional reason why we choose to work with node.js instead of php was without doubt the interestingness and relative novelty of the technology [4], which can have a substantial influence on developers. As it is unfortunate to see created artifacts fall into disuse after a project, we have decided to pay more attention to design fitness issues, as a complement to the already present focus on usefulness, in future LL-ADR projects.

\section{Conclusion}

Living Labs methods are congruous with ADR, but only to provider-driven Living Labs, where the prime focus of partners is not bringing products to the market, but generating knowledge. As ADR is a subset of DSR, the DSR literature can contribute many queues on how to conduct Living Labs as research projects. LL-ADR is a method for performing Living Lab projects by means of the Action Design Research method.

The main phases of LL-ADR are 1) Problem formulation, 2) Building, intervention, evaluation and learning (BIEL) and 3) Formalization of learning. Each phase contains a number of tasks and principles. The problem formulation phase sets the basis for the other phases and makes sure that existing knowledge on the problem class to be investigated is taken into account. The BIEL phase is iterative and generates artifacts that have an increasing level of readiness. In Living Labs, the BIEL phase will be mainly societydominant, due to the very early involvement of endusers in the design process. The Formalization of learning phase is essential for conducting a Living $\mathrm{Lab}$ as a research project. It refers to the problem formulation phase and aims to generalize what was learned during the project to the class of problems and solutions of which the artifact was an instance. As the available Living Lab methodologies do not include the formalization of learning phase, we recommend that Living Lab researchers pay more attention to it, especially in provider-driven Living Lab projects. 
Nyström et al's [10] typology of roles constitutes a useful set of roles to be used in LL-ADR projects. However, there is no design science researcher role. In future research, it would be interesting to investigate how these different roles have been instantiated in projects that followed the outlines of LL-ADR. In addition, these roles could be useful to inform society-dominant BIE in ADR projects.

Overall, this paper represents a first attempt at exaptation between the fields of DSR and Living Labs. Besides applying ADR knowledge to support Living Labs, we have discussed the application of the utility-fitness model to Living Lab research. In the future, it will be interesting to further investigate how insights from DSR can contribute to Living Labs.

Cross-fertilization in the other direction, from Living Labs to DSR is also promising. Living Lab projects mainly feature society (organization)dominant BIE and it is argued by Sein et al [15] that dominant DSR is not good at seeing artifacts as ensembles, shaped by both designers and the context of use. To Living Labs, this is the core of what distinguishes them from other approaches. Therefore, DSR may benefit from looking at how Living Lab projects handle processes that heavily influence the bottom-up social shaping of artifacts. An example of this was the crowd sourced ideation phase that was conducted as a part of the ZWERM project.

\section{References}

[1]Almirall, E., Lee, M., and Wareham, J., "Mapping living labs in the landscape of innovation methodologies", Technology Innovation Management Review, 2012. [2] Bergvall-Kareborn, B., Hoist, M., \& Stahlbrost, A. "Concept design with a living lab approach", proceedings of HICSS'09, pp. 1-10, IEEE, Hawaii, 2009.

[3] Coenen, T., Mechant, P., Laureyssens, T., Claeys, L., and Criel, J., "ZWERM: stimulating urban neighborhood self-organization through gamification", conference on Using ICT, Social Media and Mobile Technologies to Foster Self-Organisation in Urban and Neighbourhood Governance, Delft, 2013

[4] Gill, T. G., \& Hevner, A. R.. "A fitness-utility model for design science research", ACM Transactions on

Management Information Systems, 4(2), 2013

[5]Hevner, A. R., March, S. T., Park, J., \& Ram, S., "Design Science in Information Systems Research", MIS Quarterly, 28(1), pp. 75-105, 2004

[6] Järvinen, P. , "Action Research is similar to design science”, Quality \& Quantity, 41(1), pp. 37-54, 2007 [7]Laureyssens, T., Coenen, T., Claeys, L., Mechant, P., Criel, J., and Vande Moere, A., "ZWERM: a Modular Component Network Approach for an Urban Participation Game", proceedings of CHI 2014, Vancouver Canada.
[8]Leminen, S., Westerlund, M., and Nyström, A.-G.. "Living Labs as open-innovation networks. Technology", Innovation Management Review, spetember 2012 [9] Iivari, J., and Venable, J. R., “Action Research and design science research-seemingly similar but decisively dissimilar", Proceedings of 17th European Conference on Information Systems, Verona, Italy, 2009

[10] Nyström, A.-G., Leminen, S., Westerlund, M., and Kortelainen, M., "Actor roles and role patterns influencing innovation in living labs", Industrial Marketing Management, 43(3), pp. 483-495, 2014

[11] Peffers, K., Tuunanen, T., Rothenberger, M. A., and Chatterjee, S., "A design science research methodology for IS research", Journal of Management Information Systems, 24(3), pp. 45-77, 2007

[12] Pierson, J., and Lievens, B., "Configuring Living Labs For A "Thick" Understanding Of Innovation" proceedings of EPIC 2005, pp. 114-127, 2005

[13] Schaffers, H., Garcia Guzman, J., and Merz, C., "An Action Research Approach to Rural Living Labs Innovation" in Cunningham M. (Eds), Collaboration and the Knowledge Economy: Issues, Applications, Case Studies. IOS Press, 2008

[14] Schuurman, D., Baccarne, B., De Marez, L., and Mechant, P, "Smart ideas for smart cities: investigating crowdsourcing for generating and selecting ideas for ICT innovation in a City context", Journal of theoretical and applied electronic commerce research, 7(3), pp.49-62, 2012

[15] Sein, M. K., Henfridsson, O., Purao, S., Rossi, M., and Lindgren, R., Action design research. MIS Quarterly, 35(1), pp.37-56, 2011

[16] Silverstone, R., and Hirsch, E., Consuming technologies: Media and information in domestic spaces. Psychology Press, 1992

[17] Tang, T., \& Hämäläinen, M., Living lab methods and tools for fostering everyday life innovation, Proceedings of ICE Conference, pp. 1-8, IEEE, 2012

[18] Venable, J., Pries-Heje, J., \& Baskerville, R., “A comprehensive framework for evaluation in design science research", Design Science Research in IS. Advances in Theory and Practice (pp. 423-438), Springer, 2012 [19] Westerlund, M., \& Leminen, S., "Managing the challenges of becoming an open innovation company: experiences from Living Labs". TIM Review, Oktober 2011

[20] Williams, R., \& Edge, D., “The social shaping of technology”, Research Policy, 25(6), pp. 865-899, 1996 